\title{
Mathematik und Smog in Peking
}

\author{
Peter Deuflhard
}

Seit Anfang des Jahres 2013 bin ich als Professor an der Beijing University of Technology (BJUT), einer Universität der Stadt Peking. Statt der angebotenen Vollzeitstelle habe ich mich jedoch nur für wenige Aufenthalte von je ein bis zwei Wochen verpflichtet. Hauptgrund: Ich wollte mir den Smog nicht allzu lange antun, der zu dieser Zeit bereits in aller Munde war (sic!). Meine chinesischen Freunde haben mir jedoch dringend davon abgeraten, diesen Grund zu nennen, denn dabei würden meine offiziellen Gesprächspartner ,ihr Gesicht verlieren“. Zu dieser Zeit gab es einfach offiziell noch keinen Smog in Peking. Wohlgemerkt, westliche Zeitungen berichteten damals bereits ausführlich darüber und zeigten eindrucksvolle Bilder, die aber in chinesischen Medien unter die Zensur fielen.

Ein Jahr später fand wieder der alljährliche Volkskongress in Peking statt. Obwohl die Medien nichts von Smog berichteten, erfuhren jedoch die versammelten etwa 3000 Delegierten den Smog am eigenen Leib. Und sie begehrten auf! Besonders erboste sie, dass in Peking sogenannte „Smogvertilger“ nur in den Wohngegenden der politischen Elite aufgestellt waren. So kam es, dass der Ministerpräsident das Thema in seiner Abschlussrede ansprach und Besserung gelobte.

Etwa um diese Zeit erhielt ich eine Auszeichnung der Stadt Peking als sogenannter „Strategic Scientist“. Bei der Verleihung durch den Vizebürgermeister, der für Wissenschaft und Forschung zuständig war, sprach dieser zur Überraschung der Anwesenden offen davon, er hoffe, ich könne etwas für die „Umweltsituation“ in Peking tun! Wie sich herausstellte, trauten jedoch die unter ihm stehenden Kader dem neuen Trend noch nicht, sodass noch nichts richtig voranging. Man blieb lieber vorsichtig. Dennoch schien es im Frühjahr des Jahres 2014 in der mittleren politische Ebene immerhin schon formulierbar, dass es in Peking ein Problem mit dem Smog geben könnte.

Der Durchbruch kam im Frühjahr 2015: Da hielt der Staatspräsident $\mathrm{Xi}$ Jinping eine Rede, in der er die Universitäten in der Region Peking explizit aufforderte, wissenschaftlich etwas für die Verbesserung der Umweltsituation zu tun. Seit dieser Rede gab es also den Smog in Peking hochoffiziell!

In dieser Situation fühlte ich mich ermutigt, ein Projekt aufzusetzen, das mit der Drittmittelförderung aus meiner Auszeichnung finanziert werden soll. Darin soll die Umweltsituation in einem Mikroplan hinreichend detailliert mathematisch modelliert werden: Er soll die genaue Position der Verursacher des Smogs, ihre Quellstärke sowie typische Wettersituationen und Verkehrsflüsse abbilden. Die Stadt Peking, die ja Trägerin der BJUT ist, verfügt über diese Daten (verteilt über mehrere Institutio- nen) und sollte von ihrer Nutzung auch direkt profitieren. $\mathrm{Da}$ es sich bei diesen Daten um politisch sensible Daten handelt, habe ich ein NDA (non-disclosure agreement) angeregt, wie es viele Mathematiker in Industrieprojekten zu unterzeichnen gewohnt sind. Eine Smogvorhersage über einen längeren Zeitraum ist natürlich mit denselben Schwierigkeiten verbunden wie eine Wettervorhersage über einen Zeitraum von mehr als wenigen Tagen. Ziel ist stattdessen die Erarbeitung von möglichen Szenarien für politische Entscheider. Mathematisch bedeutet das, ein zeitabhängiges System partieller Differentialgleichungen für die Strömung und Diffusion in Wechselwirkung mit heterogenen chemischen Reaktionen auf der Oberfläche der Smogpartikel zu berechnen - natürlich hinreichend genau, mit Fehlerkontrolle, und am liebsten auch schnell, um möglichst viele verschiedene Szenarien durchspielen zu können.

Derzeit wird in verschiedenen Pekinger Forschungsinstitutionen der Umweltwissenschaften eine amerikanische Software zur Atmosphärenchemie eingesetzt. Diese Software realisiert eine Linienmethode, d.h. es wird zuerst im Raum, dann in der Zeit diskretisiert. Als Zeitdiskretisierung wird eine Rosenbrock-Wanner-Methode für steife Differentialgleichungen benutzt. Um die Anzahl der Unbekannten und damit die Rechenzeit in Grenzen zu halten, wird im Raum mit einer Gitterweite von ca. $3 \mathrm{~km}$ gearbeitet. Über dem Pekinger Stadtgebiet von ca. $60 \mathrm{~km} \times 60 \mathrm{~km}$ erhält man damit $(60: 3)^{2}$, also 400 horizontale Knoten. Diese Dimension ist noch zu multiplizieren mit der Anzahl vertikaler Knoten, bisher 36. Auf jedem räumlichen Knoten liegen (modellabhängig) eine Reihe von Unbekannten: Strömungsgeschwindigkeit, Temperatur, chemische Reaktanden, Verteilungsdichte der Smogpartikel.

Allerdings ist diese räumliche Diskretisierung zur Auflösung der interessierenden Phänomene viel zu grob. Um die wichtigen Prozesse - Verkehrsaufkommen, Strömung um Gruppen von Hochhäusern, Smogausstoß von Fabriken und Kraftwerken - sauber zu erfassen, sollte man an räumliche Gitterweiten von ca. $10 \mathrm{~m}$, die typische Straßen- und Häuserbreite, denken. Man rechnet also nach: $(60.000: 10)^{2}=3.6 \cdot 10^{7}$ alleine für die Anzahl horizontaler Knoten. Hiermit ist wieder die Anzahl vertikaler räumlicher Knoten möglichst adaptiv zu wählen und die Anzahl der Unbekannten zu multiplizieren. Dies führt zwingend zu Mehrgitter- und Gebietszerlegungsmethoden über adaptiven hierarchischen Raumgittern, die auf Parallelrechnern auszuführen sind - eine wissenschaftliche Herausforderung, mathematisch, algorithmisch, softwaretechnisch. Sie soll in Kooperation mit dem Kollegen Gabriel Wittum (Universität Frankfurt/KAUST) durchge- 


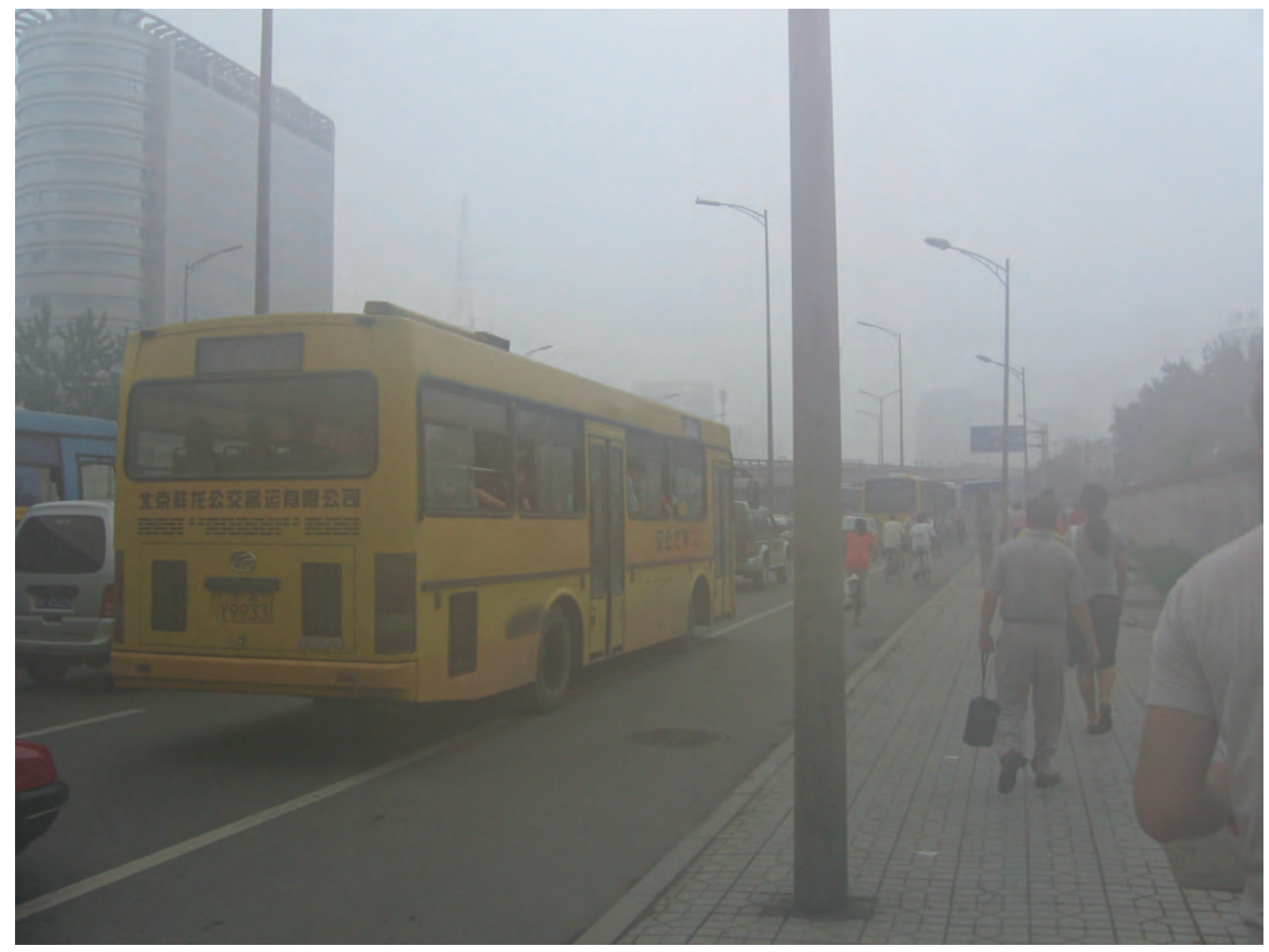

Smog in Peking (Foto: Berserkerus, CC BY-SA 3.0)

führt werden. Hinzu kommt: Da nur ein Teil der Differentialgleichungsanteile steif ist, wird man auch die natürlich adaptive Zeitdiskretisierung modifizieren wollen. Für die Modellierung entscheidend ist jedoch, auch den Verkehr als eine Ursache der Verschmutzung einzubinden, also 5 Millionen Fahrzeuge bei 22 Millionen Menschen. Dieser Teil wird vom Kollegen Rolf Möhring (TU Berlin), der ohnehin schon an Fragen der Verkehrsplanung in Peking arbeitet, in das Gesamtmodell eingespeist. Es versteht sich von selbst, dass ein solches Projekt mit Wirkung bis in die Politik hinein auch an die wissenschaftliche Visualisierung höchste Anforderungen stellen wird. Diesen Teil wird Christian Hege (ZIB) betreuen.

Es wird sicher einige Jahre dauern, bis dieses hochkomplexe Unterfangen erste Früchte tragen wird. Es soll in dem von mir gegründeten Beijing Institute for Scientific and Engineering Computing (BISEC) bearbeitet werden. Zum jetzigen Zeitpunkt ist noch ein wesentlicher Aspekt unklar: Wird es wirklich gelingen, an alle benötigten Daten zu kommen? Wie bereits oben ausgeführt, ist ein Teil dieser Daten politisch. Darüber hinaus wissen wir noch nicht, ob genügend Wissenschaftlerinnen und Wissenschaftler bei dieser Aufgabe mitmachen, weil ja nicht sofort kleinere Publikationen winken, sondern „lediglich“ ein für etwa 22 Millionen Menschen wichtiges Problem angegangen werden soll. Unsere im Schwange befindli- chen wissenschaftlichen Zählsysteme, mit denen junge Wissenschaftler oftmals eher gemessen als gewogen werden, sind in dieser Hinsicht kontraproduktiv!

Erst, wenn das gesamte Pekinger Stadtbild mit den wichtigsten Straßen, allen wesentlichen Smogerzeugern sowie dem üblichen Verkehrsaufkommen ausreichend detailliert in dem anvisierten Mikroplan erfasst sein wird und die zugehörigen zeitabhängigen partiellen Differentialgleichungssysteme schnell und korrekt gelöst werden können, erst dann gibt es den Smog in Peking auch für Mathematiker „wirklich“.

Prof. Dr. Dr. h.c. Peter Deuflhard, Zuse-Institut Berlin (ZIB), Takustraße 7, 14195 Berlin. deuflhard@zib.de

Peter Deuflhard, geb. 1944. Studium Physik, TH München 1968. Promotion Mathematik, Universität zu Köln 1972. Habilitation Mathematik, TU München 1978. Professor Numerische Mathematik, Universität Heidelberg 1978-1986. Professor Scientific Computing, Freie Universität Berlin, 1986-2014. Gründer Zuse-Institut Berlin (ZIB) 1986. Präsident ZIB 1987-2012. Mitbegründer DFG-

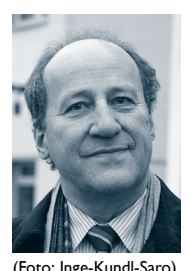

Forschungszentrum MATHEON. Seniorprofessor Sorbonne, Pierre et Marie Curie. Gründungsdirektor des Beijing Institute for Scientific and Engineering Computing (BISEC). 